\title{
Micro arrays of ring-recessed disk electrodes in transient generator-collector mode: theory and experiment
}

\author{
Denis Menshykau ${ }^{\mathrm{a}}$, Aoife M. O’Mahony ${ }^{\mathrm{a}}$, F. Javier del Campo ${ }^{\mathrm{b} *}$, \\ Francesc Xavier Muñoz ${ }^{\mathrm{b}}$, Richard G. Compton ${ }^{\mathrm{a} *}$ \\ a Department of Chemistry, Physical and Theoretical Chemistry Laboratory, \\ Oxford University, South Parks Road, Oxford, United Kingdom OX1 3QZ.

$$
\text { Fax: +44 (0) } 1865275410 \text {; }
$$ \\ bIMB-CNM. CSIC, Campus de la Universidad Autonoma de Barcelona, Bellaterra 08193, \\ Spain \\ *Corresponding authors \\ RGC: Tel: +44 (0) 1865 275413. E-mail: richard.compton@chem.ox.ac.uk \\ FJC: Tel: +34 93594 7700. E-mail: franciscojavier.delcampo@imb-cnm.csic.es
}

To be submitted as an article to:

Analytical Chemistry 


\section{Abstract}

The fabrication, characterisation and use of arrays of ring-recessed disk microelectrodes is reported. This devices are operated in generator-collector mode with a disc acting as generator and the ring as the collector. We report experiments and simulations relating to time of flight experiments in which material electrogenerated at a disc is diffusionally transported to the ring. Analysis of the current transient measured at the latter when it is potentiostatted at a value to ensure diffusionally controlled "collection" is shown to sensitively reflect the diffusion coefficients of the species forming the redox couple being driven at the generator electrode. The method is applied to the ferrocene/ferrocenium couple in the room temperature ionic liquid $\left[\mathrm{N}_{6,2,2,2}\right]\left[\mathrm{NTf}_{2}\right]$ and the results found to agree with independent measurements.

\section{Keywords}

ring-recessed disc electrode, electrode array, generator-collector electrode, bipotentiostat voltammetry, RTIL, ferrocene 


\section{Introduction}

Recently we presented a general theoretical framework to study diffusional mass transport at a new type of microelectrode array particularly suited for generator-collector experiments. ${ }^{1}$ That new geometry consisted on an array of ring-recessed disk microelectrode arrays, distributed on a planar substrate. Here we present the fabrication of these devices and demonstrate their application to the estimation of diffusion coefficients from bipotentiostatic time-of-flight experiments in ionic liquids. Time of flight experiments with electrochemical detection and generation were shown to be a general method for determination of diffusional coefficients. ${ }^{2-5}$

Disc-ring structures may be preferable to double band structures in generator-collector experiments because the generator electrode is completely surrounded by the collector electrode, so less generated material is lost by diffusion to the solution bulk, and collection efficiencies are consequently higher. In the case of microband electrodes, these losses are mostly overcome using triple band and interdigitated structures. Therefore, up until now, generator-collector experiments at microelectrodes were performed at double ${ }^{6}$ and triple ${ }^{7}$ band, or interdigitated microband structures. ${ }^{8,9}$ Regular interdigitated microbands are necessarily fabricated using microfabrication techniques based on photolithography, and their minimum geometric features are limited by the optical resolution of the photolithographic step. The most common photolithographic equipment is able to achieve resolutions of a few microns or, at most, around the micron. Electron beam lithography coupled to nanoimprint lithography allows the fabricatication of devices presenting sub-micrometric features, but these techniques are still rather uncommon due to the high cost of the required equipment and the need for very clean fabrication environments. 
In this work we demonstrate the fabrication of a new device based on disk-ring structures, using standard microfabrication techniques, and where the gap between disk and ring is below the resolution of the photolithographic step. To achieve this, the disks are fabricated recessed with respect to the rings, so the gap between them is controlled by the recess height. This height is nothing else than the thickness of a layer of dielectric material, in the present case a silicon oxide layer, that provides electrical insulation between the disks and the rings above them. Since the deposition of this dielectric layer is independent of the photolithographic step, it is possible in principle to grow very thin layers of oxide over the metal layer featuring the disks, so that nanometric scale gaps can be obtained between discs and rings, regardless of the resolution of our aligner. Another great advantage of this approach is that, since discs and rings are actually on different planes, it is possible to fabricate controlled arrays of identical disc-ring sets. This approach is not common and has never been applied for fabrication of ringdisc arrays, but has been once used to produce interdigitated array electrodes ${ }^{10,11}$ and single microcavity device with a ring-generator and tubular nanoband-collector electrode. ${ }^{12}$ This is important because although the only disc-ring microelectrodes reported to date are coplanar, the fabrication of regular arrays seems an important challenge, if not impossible, due to the way in which they are produced. ${ }^{13-16}$

In our previous work, we demonstrated that this type of device is highly efficient, and allow for steady-state collection efficiencies above $90 \%$, comparable with those obtained at interdigitated microbands. In this work we apply the diffusion domain approach used before for simulation of current responses from arrays of ring-recessed disk electrodes ${ }^{1}$ and other arrays of electrodes ${ }^{17,18}$ to aid in the determination of diffusion coefficients from potential step experiments at arrays of ring-recessed disc electrodes in generator-collector mode where 
potential on the disk is stepted from the value corresponding to no current to one of diffusion controlled electrolysis whilst the ring is potentiostated to reverse the electrolysis and convert the disk product back to starting material. Three different behaviour zones can be clearly distinguished during generator-collector experiments on fully reversible redox couples. First, at very short times, diffusion to the generator microdisc is mainly planar, because the recessed nature of the microdisc eliminates the possibility of edge diffusion to it. When the material generated by the disc reaches the ring electrode, it is converted back to the starting material and diffuses back towards the disc. After this equilibration period, a true steady-state current is achieved both at the microdisc and the microring electrodes. The duration of this so-called equilibration period is important when the diffusion coefficients of the reduced and oxidised species are different, and the current during this time is extremely sensitive to the ratio of diffusion coefficients. Another important advantage of these devices compared to conventional disc microelectrode arrays is that the steady state currents achieved are true steady state current regardless of the experiment duration, even if the array of disc-ring systems is very densely packed, since the cycling of material between disc and ring reduces the overlap of the diffusional fields of adjacent electrodes.

\section{Theoretical model}

\subsection{The model of electrode surface}

We consider an electrode with an idealized surface as shown in Figure 1. The surface is composed of an "array" of ring-recessed disk electrodes in which the disks are recessed relative to the surrounding rings. The disk electrode act as a "generator" electrode and ring electrode as 
a "collector" electrode. Material is assumed to reach the disk electrode via diffusion through the cylindrical well above it and negligibly otherwise. The diffusion of the electrochemically active species is complicated because it is intrinsically a three dimensional problem. However, the problem can be simplified by noting that each cylindrical well belongs to a diffusionally independent region known as a diffusion domain. ${ }^{17,19}$ The diffusional domain approximation treats these zones as being cylindrical with a ring-recessed disk electrode center situated at the axis of symmetry, thus reducing the problem to one of only two dimensions. The approximation is illustrated in Figure 2. Figure 2 a) and b) show the surface in Cartesian coordinates and Figure $2 \mathrm{c}$ ) identifies the unit cell in cylindrical coordinates $(r, z)$. The cylindrical radial coordinate, $r$, is defined as the distance from the axis of symmetry that runs through the center of the electrode domain. The cylindrical domain is of equal area to the hexagonal unit cell. The total current from the macroelectrode "array" is given by simple multiplication of the current from a single diffusion domain times the total number of domains $N_{\mathrm{p}}$. Recently it was shown that two dimensional simulations in conjunction with the diffusion domain approach gives results indistinguishable from full three dimensional simulations. ${ }^{20}$

\subsection{Mathematical model}

Equation 1 shows the electron transfer reaction at the generator electrode considered in these numerical simulations. Equation 2 shows the corresponding and reverse electron transfer reaction at the collector electrode. Both species A and B are assumed soluble, but only species A is assumed to be present in the bulk solution.

$$
\text { Disk : } \mathrm{A}+\mathrm{e}^{-} \rightleftharpoons \mathrm{B}
$$




$$
\text { Ring : } \mathrm{B}-\mathrm{e}^{-} \rightleftharpoons \mathrm{A}
$$

The rate of electron transfer is described by the Butler-Volmer kinetics:

$$
\left.D_{\mathrm{A}} \frac{\partial[\mathrm{A}]}{\partial z}\right|_{\mathrm{el}}=\left.\left(k_{\mathrm{f}}[\mathrm{A}]-k_{b}[\mathrm{~B}]\right)\right|_{\mathrm{el}}
$$

where $k_{\mathrm{f}}$ and $k_{\mathrm{b}}$ are given by equations 4 and 5 , consequently and $\left.D_{\mathrm{A}} \frac{\partial[\mathrm{A}]}{\partial z}\right|_{\mathrm{el}}$ is the diffusional flux of species A to the electrode surface along path Z.

$$
\begin{gathered}
k_{\mathrm{f}}=k_{0} \exp \left(-\frac{\alpha F}{R T}\left(E-E_{\mathrm{f}}^{0}\right)\right) \\
k_{\mathrm{b}}=k_{0} \exp \left(\frac{(1-\alpha) F}{R T}\left(E-E_{\mathrm{f}}^{0}\right)\right)
\end{gathered}
$$

where $E_{\mathrm{f}}^{0}$ is the formal potential of the A/B couple and $k_{0}$ is the standard rate constant of electron transfer. We next consider the potential step experiment applied to the reduction of $\mathrm{A}$ at the disk electrode and we assume that the step is big enough to assume that the concentration of $\mathrm{A}$ is zero at the disk surface. We also assume that the potential at the ring is held at a sufficiently positive value, that the concentration of B is zero at the ring. Species A and B might have different or equal diffusion coefficients. We consider both cases separately.

Equal diffusion coefficients. At each point in solution the concentration of species A and B must satisfy the mass conservation law:

$$
[\mathrm{A}]+[\mathrm{B}]=[\mathrm{A}]_{0}
$$

where $[\mathrm{A}]_{0}$ is the bulk concentration of $\mathrm{A}$. In this case the concentration profile of species $\mathrm{A}$ may be simulated independently from that of B. Mass transport in cylindrical coordinates is described by Fick's second law of diffusion given by equation 7

$$
\frac{\partial[\mathrm{A}]}{\partial t}=D_{\mathrm{A}}\left(\frac{\partial^{2}[\mathrm{~A}]}{\partial r^{2}}+\frac{1}{r} \frac{\partial[\mathrm{A}]}{\partial r}+\frac{\partial^{2}[\mathrm{~A}]}{\partial z^{2}}\right)
$$


Unequal diffusion coefficients. In case of unequal diffusion coefficients mass conservation law 6 is not fulfilled and mass transport equation 7 for species A should be accompanied by the mass transport law for the species B:

$$
\frac{\partial[\mathrm{B}]}{\partial t}=D_{\mathrm{B}}\left(\frac{\partial^{2}[\mathrm{~B}]}{\partial r^{2}}+\frac{1}{r} \frac{\partial[\mathrm{B}]}{\partial r}+\frac{\partial^{2}[\mathrm{~B}]}{\partial z^{2}}\right)
$$

Note that here we assume that the solution is fully supported ${ }^{21}$ and Fick's second law of diffusion adequately describes mass transport in fully supported media when migration can be neglected and if the length scale of the device is higher than $10 \mathrm{~nm}$.

The model is normalized with use of the dimensionless parameters which are listed in table 1. Mass transport in cylindrical coordinates is given by the dimensionless version of equations 7 and 8:

$$
\begin{gathered}
\frac{\partial a}{\partial \tau}=\frac{\partial^{2} a}{\partial R^{2}}+\frac{1}{R} \frac{\partial a}{\partial R}+\frac{\partial^{2} a}{\partial Z^{2}} \\
\frac{\partial b}{\partial \tau}=D_{\mathrm{r}}\left(\frac{\partial^{2} b}{\partial R^{2}}+\frac{1}{R} \frac{\partial b}{\partial R}+\frac{\partial^{2} b}{\partial Z^{2}}\right)
\end{gathered}
$$

The boundary conditions for equations 9 and 10 are summarized in table 2 .

\section{$3 \quad$ Experimental and computational details}

\subsection{Chemical Reagents}

N-Hexyltriethylammonium bromide (Aldrich, 99\%) was used as purchased for metathesis with lithium bis(trifluoromethyl)- sulfonylimide, and purified by standard literature procedures ${ }^{22}$ to yield $\left[\mathrm{N}_{6,2,2,2}\right]\left[\mathrm{NTf}_{2}\right]$. Ferrocene (Aldrich, 98\%), tetra-n-butylammonium perchlorate (TBAP, Fluka, Puriss electrochemical grade, $>99.99 \%$ ) and acetonitrile (Fischer Scientific, dried and distilled, >99.99\%) were used as received without further purification. 


\subsection{Instrumental}

Electrochemical experiments were performed using a computer controlled $\mu$-Autolab potentiostat (Eco-Chemie, Netherlands). A conventional two-electrode system was used for determination of the diffusion coefficient of ferrocene in $\left[\mathrm{N}_{6,2,2,2}\right]\left[\mathrm{NTf}_{2}\right]$, typically with a platinum electrode $(10 \mu \mathrm{m}$ diameter $)$ as the working electrode, and a $3.0 \mathrm{~mm}$ diameter silver wire as a quasi-reference electrode. The platinum microdisk working electrode was polished on soft lapping pads (Kemet Ltd., U.K.) using alumina powder (Buehler, IL) of size 5.0, 1.0 and $0.3 \mu \mathrm{m}$.

The electrode diameter was calibrated electrochemically by analyzing the potential-step voltammetry of a $2 \mathrm{mM}$ solution of ferrocene $(\mathrm{Fc})$ in acetonitrile containing $0.1 \mathrm{M}$ TBAP, with a diffusion coefficient for ferrocene $2.3 \times 10^{-5} \mathrm{~cm}^{2} \mathrm{~s}^{-1}$ at $298 \mathrm{~K}^{23}$

The electrodes were housed in a glass cell "T-cell" designed for investigating microsamples of ionic liquids under a controlled atmosphere. ${ }^{24,25}$ The working electrode was modified with a section of disposable micropipette tip to create a small cavity above the disk into which a drop $(20 \mu \mathrm{L})$ of ionic liquid and a drop $(20 \mu \mathrm{L})$ of $5 \mathrm{mM} \mathrm{Fc}$ in acetonitrile was placed. The RTIL solution was purged under vacuum (Edwards High Vacuum Pump, Model ES 50) for ca. 90 minutes, which served to remove trace atmospheric moisture naturally present in the RTIL, and evaporate the acetonitrile so that a concentration of $5 \mathrm{mM} \mathrm{Fc}$ remained in the RTIL. All experiments were performed inside a fume cupboard, in a thermostated box (previously described by Evans et al. $)^{26}$ which also functioned as a Faraday cage. The temperature was maintained at $298( \pm 1.0) \mathrm{K}$.

An array of ring-recessed disk electrodes was used to compare the theoretical generator/collector model outline in Section2.2 with experimental data. The electrode array was 
rinsed well with water and dried with nitrogen. The Fc/[ $\left.\mathrm{N}_{6,2,2,2}\right]\left[\mathrm{NTf}_{2}\right]$ solution used at the platinum electrode was removed from the cavity above the disk using a micropipette and dropped onto the surface of the electrode array. Experiments were performed in a faraday cage at room temperature.

\subsection{Microdisc and Micro Array Chronoamperometric Experiments}

Chronoamperometric transients at the platinum electrode $(10 \mu \mathrm{m}$ diameter $)$ were achieved using a sample time of $0.01 \mathrm{~s}$. After pre-equilibration for 20 seconds, the potential was stepped from a postion of zero current to a chosen potential after the oxidative peak of ferrocene, and the current was measured for $5 \mathrm{~s}$. The software package Origin 7.0 (Microcal Software Inc.) was used to fit the experimental data. The equations proposed by Shoup and $\mathrm{Szabo}^{27}$ (below) were imported into the non-linear curve fitting function.

$$
\begin{gathered}
I=-4 n F D c r_{\mathrm{d}} f(\tau) \\
f(\tau)=0.7854+0.4432 \tau^{-\frac{1}{2}}+0.2146 \exp \left(-0.3912 \tau^{-\frac{1}{2}}\right)
\end{gathered}
$$

where $n$ is the number of electrons transferred, $F$ is the Faraday constant, $D$ is the diffusion coefficient, $[\mathrm{A}]_{0}$ is the initial concentration of parent species, $r_{0}$ is the radius of the disk electrode, and $\tau$ is the time. The equations used in this approximation are sufficient to give $D$ and $c$ within an error of $0.6 \%$.

The value for the radius (previously calibrated) was fixed, and a value for the diffusion coefficient and the product of the number of electrons and concentration was obtained after optimization of the experimental data.

Double potential step chronoamperometric transients at the platinum electrode $(10 \mu \mathrm{m}$ 
diameter) were achieved using a sample time of $0.01 \mathrm{~s}$. The solution was pretreated by holding potential at a point of zero current for $20 \mathrm{~s}$, after which the potential was stepped to a position after the oxidative peak for Fc, and the current was measured for $5 \mathrm{~s}$. The potential was then stepped back to a point of zero current, and the current response measured for a further 5 s. In order to extract diffusion coefficients from these transients, the first potential step was fitted as before using the a nonlinear curve fitting function in the software package Origin 7.0 (Microcal Software Inc.) following eqns 11 and 12 as proposed by Shoup and Szabo. To model the second potential step, a computer simulation program (described by Klymenko et al.) ${ }^{28}$ was employed. Values of $D$ and $[\mathrm{A}]_{0}$ obtained from Shoup and $\mathrm{Szabo}^{27}$ analysis of experimental data were input into simulation software and values of $D$ for the reverse step were varied until the best fit between theoretical and experimental data was achieved.

Bipotentiostat chronoamperometry was performed on the ring-recessed disk electrode array to oxidise and reduce Fc. The microdisk array was used as a generator electrode to oxidise Fc to $\mathrm{Fc}^{+}$, and the microring array was used as the collector electrode to reduce $\mathrm{Fc}^{+}$to $\mathrm{Fc}$. The solution was pretreated by holding potential at a pont of zero current for $20 \mathrm{~s}$, after which the potential on the microdisk array was stepped to potential after the oxidative peak for Fc. The current was measured for $0.5 \mathrm{~s}$ at a sample time of $0.001 \mathrm{~s}$, and then for $20 \mathrm{~s}$ at a sample time of $0.004 \mathrm{~s}$. Simultaneously the potential was stepped back to a point after the reduction of $\mathrm{Fc}^{+}$ to Fc on the microring array. The current was measured under the same conditions as that of the microdisk array. 


\subsection{Array fabrication procedure}

Microelectrode arrays were fabricated using standard photolithographic techniques as follows.

A 1 micron thick layer of thermal oxide was grown on 4-inch diameter silicon wafers to provide electrical insulation to the microelectrodes (Figure 3.1). Next the bottom metal layer was deposited by sputtering (Figure 3.2). This metal layer consisted of $25 \mathrm{~nm} \mathrm{Ti}, 25 \mathrm{~nm} \mathrm{Ni}$ and $125 \mathrm{~nm} \mathrm{Au}$. The Titanium was used to promote adhesion of the gold over the silicon oxide. Nickel was used as a diffusion barrier to avoid contamination of the gold by titanium due to the high temperatures achieved during sputtering. Next, a positive photoresist was deposited by spin coating over the gold. This photoresist was developed after contact-UV insolation through a clear field mask (Figure 3.a). Following this, the bottom metal areas and the contacts were defined in a wet-etching step (Figure 3.3). The excess photoresist was stripped once these patterns had been defined, and a thin silicon oxide layer (ca. 1 micron) was grown over the wafers (Figure 3.4). This oxide layer provided electrical insulation and provided the separation between the discs and the rings in the final device. Following the deposition of the intermediate oxide layer, a second metal layer was deposited and patterned in the same way as the previous one (Figure 3.5-6). The only special feature of this second metal layer was that it featured "holes" corresponding to the underlaying microdiscs (Figure 3.b). This way the top gold layer will also be used as mask during the final etching steps. Following this second metallisation, a passivation layer was deposited over the wafers to provide electrical insulation to the devices. This passivation layer consisted of $200 \mathrm{~nm}$ of silicon oxide and $400 \mathrm{~nm}$ of silicon nitride (Figure3.7).

The next steps of the fabrication were critical, as it involved opening the contacts and 
defining the rings and discs in the top and bottom metal levels, respectively.

A positive photoresist was spin coated over the surface of the nitride face of the wafers. A dark field chromium mask featuring disks was used to pattern the microrings on the top metal layer (Figure 3.c). After the photoresist was developed, the silicon nitride and oxide over the top metal layer was etched by reactive ion etching, RIE. During this step, the top metal layer served as mask and thus the intermediate oxide layer between metal levels could also be etched (Figure 3.8). The trouble with this approach is that the RIE may damage the gold and therefore the time of attack must be carefully controlled in order not to "burn" the top metal level. As a result of this and also of the fact that the different passivating layers present slight inhomogeneities depending on the position of the wafer in various ovens, intermediate silicon oxide is not successfully removed from all the disc-ring systems, and hence there are a low number of active microdiscs compared to more common microelectrode arrays. After the RIE step, the excess resin was stripped in acetone and the wafers thoroughly rinsed in deionised water. The wafers were then diced into individual chips, which were subsequently attached, wire-bonded and encapsulated on suitable print circuit board strips.

\subsection{Computational procedure}

Because of the symmetry of the model, the mass transport equations 7 and 8 and its accompanying conditions (table 2 ) were solved in the two dimensional space in the region $0<R<R_{\max }$ and $Z>0$. The bulk solution condition is implemented at a distance $6 \sqrt{\tau_{\max } * \max \left\{1, D_{\mathrm{r}}\right\}}$ from the highest point of the electrode, where $\tau_{\max }$ is the time scale of experiment. Beyond this the effects of diffusion are not important on the experimental voltammetric timescale. ${ }^{29,30}$ The modeling of the ring-recessed disk electrode is complicated by the presence of singularities at 
the boundaries between electrodes and insulators. To calculate the precise values of the current at the electrodes a rectangular expanding grid, similar to that used in previous simulations of electrodes with complicated surfaces ${ }^{31-34}$ was utilised. The expanding grid is defined by equations 13-16 and the schematic view of the grid is presented in figure 4 .

$$
\begin{aligned}
& R_{\mathrm{i}+1}-R_{\mathrm{i}}=h_{\mathrm{i}} \\
& Z_{\mathrm{i}+1}-Z_{\mathrm{i}}=k_{\mathrm{i}} \\
& h_{\mathrm{i}}=\gamma_{\mathrm{R}} \times h_{\mathrm{i}-1} \\
& k_{\mathrm{i}}=\gamma_{\mathrm{Z}} \times k_{\mathrm{i}-1}
\end{aligned}
$$

Furthermore if the distance separating the disk from the ring is small, very fine time steps are required to calculate accurate values of current. Standard values of the mesh used in computations were $h_{0}=k_{0}=10^{-5}$ and $\gamma_{\mathrm{R}}=\gamma_{\mathrm{Z}}=1.125$. An expanding time grid is also used; time steps were calculated each time in agreement with the expression:

$$
\min \left(\tau_{\mathrm{k}} \tau^{\mathrm{m}}, \gamma_{\tau} \delta \tau\right)
$$

where, standard values of parameters are $\tau_{\mathrm{k}}=0.001, m=0.65, \gamma_{\tau}=1.01$ and initial time step is $\delta \tau=10^{-10}$. The alternating direction implicit finite difference method ${ }^{35}$ was used in conjunction with the Thomas algorithm ${ }^{36}$ to solve the discretized form of the mass transport equations 9 and 10 . The program was written in $\mathrm{C}++$.

The dimensionless current at a generator and collector electrodes of cylindrical symmetry was calculated with formula 18 and 19 respectively.

$$
j=\frac{\pi}{2} \int_{0}^{1} j^{\prime} R \mathrm{~d} R
$$




$$
j=\frac{\pi}{2} \int_{1}^{R_{1}} j^{\prime} R \mathrm{~d} R
$$

$j^{\prime}=\frac{\partial a}{\partial Z}$ was calculated with a three point approximation. ${ }^{37}$

\section{Results and Discussions}

\subsection{Isolated Ring-Recessed Disk Electrodes: Theory}

Equal diffusional coefficients. Figure 5 shows current transients and concentration profiles calculated on ring-recessed disk electrodes. At short times $\left(\tau<10^{-4}\right)$ diffusion to the generator electrode (disk) is planar (Figure $5 \mathrm{~b}$ ) and the current corresponds to that predicted by the Cottrell equation; no current is at that time observed on the ring (generator) electrode. At $\tau>$ $10^{-3}$ the electrogenerated species B reaches the collector electrode (Figure $5 \mathrm{c}$ ) and an increase of current on it is observed. However, the diffusion to the collector electrode is still planar and the current scales inversely with square root of time. With further increase of time the thickness of the diffusional layer increases (Figure $5 \mathrm{~d}$ )) and the current on the collector electrode grows. Current on the generator electrode is not described by the Cottrell equation anymore but decays slowly because of the convergent diffusion. At very long times $\left(\tau>10^{3}\right)$ steady state currents are observed at both generator and collector electrodes and the concentration profile does not changes over the the time (Figure 5 e).

Figure 6 shows the influence of the cylinder depth $L_{\text {cyl }}$ on the current transients on the generator and collector electrodes. An increase of ring to disc separation causes an increase of time scale for which the generator electrode behaves as a macroelectrode. This is because the sides of the well surrounding the disc constrain the diffusion to being one dimensional, 
similar behavior was observed before on the disc recessed electrodes. ${ }^{20,38-40}$ At the same time increase of the parameter $L_{\text {cyl }}$ causes increased delay of the current appearing at the collector electrode. Increasing the cylinder depths/recess heights have a decreasing effect on the steady state current both on generator and collector electrodes.

Unequal Diffusion Coefficients. Figure 7 shows current transients calculated for the case of unequal diffusional coefficients. Current transitions on both generator and collector electrodes is sensitive to the ratio of the diffusion coefficients $D_{\mathrm{r}}$. If the diffusion coefficient of the reduced species $\mathrm{B}$ is less than that of the initial form $\mathrm{A}$, then the current at the generator electrode will show a "delay" compared with the case of equal diffusion coefficients. The current on the generator electrode goes through a minimum and before it reaches the steady state value if the diffusion coefficient of reduced species $B$ is less than that of the initial form. If the diffusion coefficient of the reduced form is higher than that of oxidised then it reaches collector electrode faster than in case of equal diffusion coefficients. Consequently current at the collector electrode rises faster (time of flight $\sim L_{\text {cyl }}^{2} / D_{\mathrm{r}}$ ). However supply of electroactive species to generator electrode is limited by the slow diffusion of oxidised form (time of flight $\sim L_{\text {cyl }}^{2}$ ). Slow generation of reduced specie on the disc electrode causes a decrease of current on collector electrode, consequently the current on it goes through the maximum until the steady state is reached. The value of the steady state current on both generator and collector electrode depends on the geometry of the ring-disk electrode and on the diffusion coefficient of the initial species A, but is not affected by the value of the diffusion coefficients of reduced species $\mathrm{B}\left(D_{\mathrm{B}}\right)$. 


\subsection{Effect of the Array Density}

Figure 8 shows the influence of the diffusion domain radius on the current transients. It is clear that the current on the generator electrode is almost independent of the diffusion domain radius. This is contrary to the behavior of a flat array of microelectrodes (in the absence of the collector) where the current dramatically depends on the diffusional domain radius ${ }^{41}$ and timescale of the experiment. It was shown that the nano or microelectrode in the array with any separation of electrodes within the array can be assumed to be diffusionally independent, only for limited time, after which the array as a whole behaves as a macroelectrode ${ }^{41}$ (see solid curves on figure 8). This dramatic difference can be understood in terms of the concentration profiles presented in figure 9. This shows the concentration profile for an analite at flat microelectrode array and also for ring-recessed disk electrode. The size of the diffusional layer on the array of flat microelectrodes increases with time, however on the generator-collector system it increases significantly only at very short times, which makes the microelectrodes in the array diffusionally independent at all but the very longest timescales of the experiment. In table 3 duration and values of the steady state currents from an array of microdisks and an array of generator collector electrodes are presented. It is clear that the generator-collector electrodes have greater advantages over the ordinary arrays of microdisks when the radius of the electrode is small as can be judged from table 3 . At these values of the radius, the duration of steady state currents on array of microelectrodes is too short, however on arrays of the generator-collector electrodes due to the diffusional independence of electrodes within the array the current does not deviate from the steady state value over time. Furthermore use of generator-collector electrodes allows dense packing of electrodes in the array without loss of the duration of steady state current. 


\subsection{Array Characterisation}

Figure 10 shows images of the fabricated array obtained to confirm its geometry. The ringrecessed disc microelectrode arrays were characterised by perfilometry using a P15 KLA Tencor instruments perfilometer mounting a low force head. The tip used was a $2 \mathrm{~mm}$ diamond stylus tip with a 60 cone angle. These measurements allowed the determination of the various passivation layers. The height of the top nitride and oxide passivation layer was $710+/-50 \mathrm{~nm}$, and the disc recess height relative to the rings was 1.50 microns Figure 10 a. In addition, confocal microscopy was also used to obtain a 3D optical images of the devices shown in Figure 10 b. For these measurements, a PL $\mu$ non-contact confocal imaging profiler system attached to a Nikon microscope using a 50x magnification lens was employed, and controlled using PL $\mu$ proprietary software (Sensofar, Spain). The same software was used to analyse the images and extract topological data. Confocal measurements were in good agreement with perfilometric measurements. Optical microscopy was also used to verify the diameter of the discs and rings making up the arrays, which were 10 microns for disk diameter and 30 microns for ring diameter (Figure $10 \mathrm{~b}$ ). Figure $10 \mathrm{c}$ shows the whole array, the number of ring-recessed disc electrodes in the array is 130 .

\subsection{Experimental Validation}

Diffusion coefficients measurements. Figure 11a shows a cyclic voltammogram of the oxidation and reduction of ferrocene $(\mathrm{Fc})$ and ferrocenium $\left(\mathrm{Fc}^{+}\right)$respectively in $\left[\mathrm{N}_{6,2,2,2}\right]\left[\mathrm{NTf}_{2}\right]$ at 100 $\mathrm{mV} \mathrm{s}^{-1}$ at $10 \mu \mathrm{m}$ diameter Pt electrode the solution was purged under vacuum for 90 min. Ferrocene is oxidized by one-electron to ferrocenium at a peak potential of $+0.14 \mathrm{~V}$ vs $\mathrm{Ag}$, and 
reduced back to ferrocene at a peak potential of $+0.05 \mathrm{~V}$ vs Ag, corresponding to eqn 20 .

$$
\left[\mathrm{Fe}\left(\mathrm{C}_{5} \mathrm{H}_{5}\right)_{2}\right] \rightleftharpoons\left[\mathrm{Fe}\left(\mathrm{C}_{5} \mathrm{H}_{5}\right)_{2}\right]^{+}+\mathrm{e}^{-}
$$

Double potential step chronoamperometry was carried out to calculate diffusion coefficients of Fc and its oxidized species, $\mathrm{Fc}^{+}$. The potential was stepped from $-0.1 \mathrm{~V}$ to $+0.4 \mathrm{~V}$ to oxidise $\mathrm{Fc}$ to $\mathrm{Fc}^{+}$, and then stepped back to $-0.1 \mathrm{~V}$ to reduce $\mathrm{Fc}^{+}$to $\mathrm{Fc}$. The transient obtained is shown as the solid line in Figure 11. The diffusion coefficient, D and concentration, c of neutral Fc was determined from analysis of the experimental data for the first potential step using Shoup and $\mathrm{Szabo}^{27}$ approximations. A computer simulation program ${ }^{28}$ was used to model the second potential step to give $\mathrm{D}_{\mathrm{Fc}^{+}}$. The value for $\mathrm{D}_{F c}$ was calculated to be $10.0 \times 10^{-12} \mathrm{~m}^{2} \mathrm{~s}^{-1}$ which is consistent with previous observations reported for other RTILs. ${ }^{26,42}$ The value for $\mathrm{D}_{\mathrm{Fc}^{+}}$was calculated to be $5.50 \times 10^{-12} \mathrm{~m}^{2} \mathrm{~s}^{-1}$. The ratio $\mathrm{D}_{F c} / \mathrm{D}_{F c^{+}} \neq 1$, suggesting that the neutral $\mathrm{Fc}$ species diffuses more quickly than the charged $\mathrm{Fc}^{+}$species through the ionic liquid media, an effect similar to that observed for oxygen/superoxide in RTIL $^{43}$

The same Fc/RTIL solution used to determine the above diffusion coefficient and concentration values, was dropped onto the electrode array surface. The solution had previously been purged under vacuum for $90 \mathrm{~min}$. and was used without further degassing.

Generator collector mode. The array was immersed in a solution of Fc of known concentration. Figure 12 shows a bipotentiostat chronoamperometric transients using the microdisk array as the generator and the microring array as the collector. The potential on the microdisk array was stepped from $-0.1 \mathrm{~V}$ to $+0.4 \mathrm{~V}$ to oxidise $\mathrm{Fc}$ to $\mathrm{Fc}^{+}$and potential on the microring array was kept at $-0.1 \mathrm{~V}$ to reduce $\mathrm{Fc}^{+}$to $\mathrm{Fc}$. The transients obtained are shown as the solid line in Figure 12. The diffusion coefficients of $\mathrm{Fc}_{\mathrm{c}}$ and $\mathrm{Fc}^{+}$and the concentration of the $\mathrm{Fc}$ in 
solution have been previously determined at the platinum electrode and are known.

To model the transient at generator and collector electrode, diffusion coefficients and concentration values were used as determined in double step chronoamperometry shown in figure 11. The geometry of the array were used as determined from microscopy (figure 10) and the number of active generator-collector electrodes $N$ were varied. The optimised value of active generator-collector microelectrodes was $N=26$. The fraction of the active electrodes is $20 \%$ which is less than the typical value of active electrodes in conventional microdisk electrodes arrays. ${ }^{41,44}$ This is due to deficiencies in the final etching step of the fabrication process. Presumably, due to slight inhomogeneities in oxide layer thicknesses, the reactive ion etching has not been able to open all the microdisks and hence the low active number. To show that the theoretical model presented above adequately describes fabricated arrays of the generatorcollector electrodes in the presence of dead devices we consider different types of dead devices. Devices with an inactive ring and an inactive disc do not contribute to the recorded current and they do not distort current transients from neighbouring electrodes due to diffusional independence of the generator-collector system (Figure 8); devices with a dead disc and an active ring do not generate any current due to absence of electroactive species at the ring electrode; finally devices with an active disc only can distort the current, but voltammograms and current transients recorded at ring only and disc only modes suggest that the number of active rings is significantly higher than number of active discs, consequently the presence of such a devices is negligible. It is clear that the theoretical fit is in good agreement with the experimental data. Particularly, the theoretical fit represents well one-dimensional diffusion to microdisk at short times when the $\mathrm{Fc}^{+}$has not yet reached the collector electrode (Figure $9 \mathrm{~b}$ ). It also describes well the region of times where diffusion is converged and $\mathrm{Fc}^{+}$reduced to $\mathrm{Fc}$ at the collector 
electrode and diffuses back to the generator (Figure $9 \mathrm{~d}$ and $\mathrm{f}$ ). The fit predicts the feature that current transient on the generator electrode goes through the minima and finally it gives accurate values of steady state current on generator and collector electrodes. This shows that two dimensional simulations in conjunction with the diffusion domain approach gives accurate description of a full three dimensional problem of mass transport at ring-recessed disk electrodes, taking into account sensitivity of current transients, particularly the depth of minima/maxima and the time of flight to the ratio of diffusion coefficients (Figure 7). Consequently we conclude that the method can be applied to simultaneous determination of diffusion coefficients of red/ox couple.

\section{Conclusions}

Three different time regimes are observed in a potential step experiment on ring-recessed disk electrode. First a rapid decrease of current on generator similar to that on macroelectrode and no-current on the collector is observed at short times. This is followed by the region where current at the collector grows and current at the generator "equilibrates". Finally a true steady state current is observed on both generator and collector electrodes. All three regions of behavior were observed experimentally on a fabricated array of generator-collector array. The theoretical fit agrees well with the experimental data, therefore diffusional domain approach can be used to model arrays of ring-recessed disc electrodes. Currents in the steady state mode are almost independent of the distance between electrodes in the array, but sensitive to the distance between disk and ring $L_{\text {cyl }}$. Steady state currents is also insensitive to the ratio of diffusional coefficients, but the modeling of current transients on the generator and collector 
electrodes allows the determination of diffusion coefficients to both oxidised and reduced forms simultaneously. The use of arrays of ring-recessed disk electrodes in generator-collector mode has advantages over conventional arrays of disk electrodes, such as higher currents at a single microelectode in an array. The number of electrodes on the array can also be increased, as smaller interelectrode distances are possible. As well as this, the disks in the array are not restricted by size as true steady-state behavior is observed over a wide range of scales.

\section{Acknowledgment}

D.M. thanks St. John's College, Oxford, for a Kendrew Scholarship, A.O.M. thanks Honeywell Analytics for financial support and JdC acknowledges Ramon y Cajal Fellowship from the Spanish Ministry of Science and Innovation. 


\section{References}

[1] Menshykau, D.; Javier del Campo, F.; Munoz, F.; Compton, R. Sens. Actuators, B 2009, 138, 362-367.

[2] Wittek, M.; Mller, G.; Johnson, M.; Majda, M. Analytical Chemistry 2001, 73(5), 870-877.

[3] Amatore, C.; Sella, C.; Thouin, L. Journal of Physical Chemistry B 2002, 106(44), 1156511571.

[4] Slowinska, K.; Feldberg, S.; Majda, M. Journal of Electroanalytical Chemistry 2003, 554$555(1), 61-69$.

[5] Amatore, C.; Sella, C.; Thouin, L. Journal of Electroanalytical Chemistry 2006, 593(1-2), 194-202.

[6] Fosset, B.; Amatore, C.; Bartelt, J.; Michael, A.; Wightman, R. Anal. Chem. 1991, 63, 306-314.

[7] Fosset, B.; Amatore, C.; Bartelt, J.; Wightman, R. Anal. Chem. 1991, 63, 1403-1408.

[8] Niwa, O.; Morita, M.; Tabei, H. Anal. Chem. 1990, 62, 447-452.

[9] Aoki, K.; Morita, M.; Niwa, O.; Tabei, H. J. Electroanal. Chem. 1988, 256, 269-282.

[10] Niwa, O.; Morita, M.; Tabei, H. August 1989, 267(1-2), 291-297.

[11] Ivanic, R.; Rehacek, V.; Novotny, I.; Breternitz, V.; Spiess, L.; Knedlik, C.; Tvarozek, V. May 2001, 61(2-4), 229-234.

[12] Vandaveer IV, W. R.; Woodward, D. J.; Fritsch, I. September 2003, 48(20-22), 3341-3348. 
[13] Zhao, G.; Giolando, D. M.; Kirchhoff, J. R. Anal. Chem. 1995, 67, 1491-1495.

[14] Liljeroth, P.; Johans, C.; Slevin, C. J.; Quinn, B. M.; Kontturi, K. Electrochem. Comm. 2002, 4, 67-71.

[15] Harvey, S. L. R.; Parker, K.; O’Hare, D. J. Electroanal. Chem. 2007, 610, 122-130.

[16] Harvey, S. L. R.; Coxon, P.; Bates, D.; Parker, K. H.; O'Hare, D. Sens. Actuators B 2008, 129, 659-665.

[17] Amatore, C.; Sav́eant, J. M.; Tessier, D. J. Electroanal. Chem. 1983, 147, 39-51.

[18] Davies, T. J.; Moore, R. R.; Banks, C. E.; Compton, R. G. J. Electroanal. Chem. 2004, 574, 123-152.

[19] Brookes, B. A.; Davies, T. J.; Fisher, A. C.; Evans, R. G.; Wilkins, S. J.; Yunus, K.; Wadhawan, J. D.; Compton, R. G. J. Phys. Chem. B 2003, 10\%, 1616-1627.

[20] Guo, J.; Lindner, E. Anal. Chem. 2009, 81, 130-138.

[21] Dickinson, E. J. F.; Limon-Petersen, J. G.; Rees, N. V.; Compton, R. G. J. Phys. Chem. C 2009, 113, 11157-11171.

[22] Bonhôte, P.; Dias, A.-P.; Papageorgiou, N.; Kalyanasundaram, K.; Grätzel, M. Inorg. Chem. 1996, 35, 1168-1178.

[23] Sharp, M. Electrochim. Acta 1983, 28, 301-308.

[24] Schröder, U.; Wadhawan, J. D.; Compton, R. G.; Marken, F.; Suarez, P. A. Z.; Consorti, C. S.; de Souza, R. F.; Dupont, J. New J. Chem. 2000, 24, 1009-1015. 
[25] Silvester, D. S.; Aldous, L.; Hardacre, C.; Compton, R. G. J. Phys. Chem. B 2007, 111, $5000-5007$.

[26] Evans, R. G.; Klymenko, O. V.; Price, P. D.; Davies, S. G.; Hardacre, C.; Compton, R. G. ChemPhysChem 2005, 6, 526-533.

[27] Shoup, D.; Szabo, A. J. Electroanal. Chem. 1982, 140, 237-245.

[28] Klymenko, O. V.; Evans, R. G.; Hardacre, C.; Svir, I. B.; Compton, R. G. J. Electroanal. Chem. 2004, 571, 211-221.

[29] Svir, I. B.; Klymenko, O. V.; Compton, R. G. Radiotekhnika 2001, 118, 92-101.

[30] Bard, A. J.; Faulkner, L. R. Electrochemical Methods: Fundamentals and Application; John Wiley and Sons: New York, 2001.

[31] Gavaghan, D. J. J. Electroanal. Chem. 1998, 456, 1-12.

[32] Dickinson, E. J. F.; Streeter, I.; Compton, R. G. J. Phys. Chem. B 2008, 112, 4059-4066.

[33] Dickinson, E. J. F.; Streeter, I.; Compton, R. G. J. Phys. Chem. C 2008, 112, 1163711644.

[34] Davies, T. J.; Compton, R. G. J. Electroanal. Chem. 2005, 585, 63-82.

[35] Peaceman, J.; Rachford, H. J. Soc. Ind. Appl. Math. 1955, 3, 28-41.

[36] Atkinson, K. Elementary Numerical Analysis, 3rd ed; John Wiley and Sons: New York, 2004.

[37] Gavaghan, D. J. J. Electroanal. Chem. 1997, 420, 147. 
[38] Amatore, C.; Oleinick, A. I.; Svir, I. November 2006, 597(1), 77-85.

[39] Amatore, C.; Oleinick, A. I.; Svir, I. June 2009, 81(11), 4397-4405.

[40] Menshykau, D.; Compton, R. G. February 2009, 25(4), 2519-2529.

[41] Menshykau, D.; Huang, X.-J.; Rees, N. V.; Del Campo, F. J.; Munoz, F.; Compton, R. G. Analyst 2009, 134, 343-348.

[42] Rogers, E. I.; Silvester, D. S.; Poole, D. L.; Aldous, L.; Hardacre, C.; Compton, R. G. J. Phys. Chem. C 2008, 112, 2729-2735.

[43] Evans, R. G.; Klymenko, O. V.; Saddoughi, S. A.; Hardacre, C.; Compton, R. G. The Journal of Physical Chemistry B 2004, 108, 7878-7886.

[44] Ordeig, O.; Banks, C. E.; Davies, T. J.; Del Campo, J.; Mas, R.; Munoz, F. X.; Compton, R. G. Analyst 2006, 131, 440-445. 


\section{List of figures}

Figure 1. Schematic diagram of the electrode surface.

Figure 2(a)Schematic diagram of the unit cell for an array of ring-recessed electrodes; (b) single unit cell in Cartesian coordinates; (c) equivalent diffusion domain in cylindrical coordinates.

Figure 3. Diagrammatic representation of main steps in the fabrication process (1-8) and mask types used (a-c).

Figure 4 Expanding grid used in this work (some lines excluded for clarity). The generator electrode is shown in solid black, the collector electrode is in black squares and the insulator is in black dashes.

Figure 5. a) Calculated dimensionless current at generator and collector electrodes (solid lines) versus dimensionless time in the potential step experiment, dashed line represents current transient given by the Cottrell equation, $L_{\mathrm{cyl}}=0.1$. b), c), d), e) Concentration profile at $\tau=10^{-4}, 10^{-3}, 0.1,100$ correspondingly

Figure 6. Absolute value of calculated dimensionless current at generator a) and collector b) versus dimensionless time in the potential step experiment, single electrode, $R_{1}=1.1$. Solid and dotes lines corresponds to current at macro- and microelectrodes. Dashed lines represents current at recessed generator-collector electrodes with $L_{\mathrm{cyl}}=0.01 \mathrm{a}, 0.032 \mathrm{~b}, 0.1 \mathrm{c}, 0.32 \mathrm{~d}, 1 \mathrm{e}$, $3.2 \mathrm{f}, 10 \mathrm{~g}, 32 \mathrm{~h}$.

Figure 7. Absolute value of calculated dimensionless current at generator a) and collector b) electrodes versus dimensionless time in the potential step experiment, single electrode, $R_{1}=1.1$, $L_{\text {cyl }}=0.5$. Dashed line represents case of equal diffusion coefficients, solid lines represents 
results of modeling with unequal diffusion coefficients. $D_{\mathrm{r}}$ have values of $0.1,0.16,0.25,0.40$, $0.63,1.6,2.5,4.0,6.3,10$ from the lowest to the highest line.

Figure 8. Influence of diffusional domain radius on current transients. $R_{1}=1.1$ and $L_{\text {cyl }}=$ 0.1. a) circles corresponds to single microelectrode, solid line h corresponds to macroelectrode, solid lines $\mathrm{g}, \mathrm{f}$ and e corresponds to microelectrodes in the array with $R_{\max }=5,10$ and 13 correspondingly, dashed lines a and b, c, d represents current on generator at single recessed generator-collector device and the array of generator-collector device with $R_{\max }=10,5$ and 2 correspondingly. b) dashed lines a and b, c, d represents current on collector at single recessed generator-collector device and the array of generator-collector device with $R_{\max }=10,5$ and 2 correspondingly.

Figure 9. Simulated concentrations profiles, at array of microelectrodes (a), c), e), g)) and recessed generator-collector (b), d), f), h)): $\tau=0.001$ a) and b), $\tau=0.1$ c) and d), $\tau=10$ e) and $\mathrm{f}), \tau=100 \mathrm{~g})$ and $\mathrm{h}$ ).

Figure 10. Perfolimetry profile. b) Confocal microscopy image showing, in detail, a typical ring-recessed disc electrode in an array. The disc diameter, which is also the ring inner diameter, is 10 microns. The ring outer diameter is 30 microns. The disc recess height is approximately 1.6 microns c) Optical microscope image of the chip type used in this work. The chip features 4 electrodes. From left to right, 1 is a large electrode that can be used as auxiliary electrode. 2 corresponds to the recessed microdiscs, 3 is a thin microband intended for use as quasi-reference electrode and 4 connects to the top microring electrodes.

Figure 11. a) Cyclic voltammogram of ferrocene in $\left[\mathrm{N}_{6,2,2,2}\right]\left[\mathrm{NTf}_{2}\right]$ at $10 \mu \mathrm{m}$ Pt electrode vs $\mathrm{Ag}$ wire at $100 \mathrm{mV} \mathrm{s}^{-} 1$. b)Double-step chronoamperometry of Fc in $\left[\mathrm{N}_{6,2,2,2}\right]\left[\mathrm{NTf}_{2}\right]$ at microdisk platinum electrode $(10 \mu \mathrm{m}$ diameter $) v s$ silver wire for duration $5 \mathrm{~s}$ at sample time 
$0.01 \mathrm{~s}$. Experimental data is shown as a line and theoretical fit is plotted as cycles.

Figure 12. Bipotentiostat chronoamperometry of $\mathrm{Fc}$ in $\left[\mathrm{N}_{6,2,2,2}\right]\left[\mathrm{NTf}_{2}\right]$ at ring-recessed disk electrode array for a) generator electrode and b) collector electrode, experimental data is shown as a line and theoretical fit is plotted as cycles. $\mathrm{D}_{\mathrm{Fc}}=10.0 \times 10^{-12}, \mathrm{D}_{\mathrm{Fc}^{+}}=5.50 \times 10^{-12}$. 


\section{Tables}

Table 1: Dimensionless parameters used for numerical simulation.

\begin{tabular}{|c|c|}
\hline Parameter & Expression normalized to $r_{0}$ \\
\hline Radial coordinate & $R=r / r_{0}$ \\
\hline Normal coordinate & $Z=z / r_{0}$ \\
\hline Time & $\tau=\frac{D t}{r_{0}^{2}}$ \\
\hline Scan rate & $\sigma=\frac{F v r_{0}^{2}}{R T D}$ \\
\hline Potential & $\theta=\frac{F\left(E-E_{\mathrm{f}}^{0}\right)}{R T}$ \\
\hline Concentration of species A & $a=[\mathrm{A}] /[\mathrm{A}]_{0}$ \\
\hline Concentration of species B & $b=[\mathrm{B}] /[\mathrm{A}]_{0}$ \\
\hline Pore depth & $L_{\mathrm{cyl}}=\frac{z_{0}}{r_{0}}$ \\
\hline Outer radius of collector ring & $R_{1}=\frac{r_{1}}{r_{0}}$ \\
\hline Radius of diffusion domain & $R_{\max }=\frac{r_{\max }}{r_{0}}$ \\
\hline Diffusion coefficient ratio & $D_{\mathrm{r}}=\frac{D_{\mathrm{B}}}{D_{\mathrm{A}}}$ \\
\hline Electrode current & $j=\frac{-i}{4 F D[\mathrm{~A}]_{0} r_{0}}$ \\
\hline
\end{tabular}


Table 2: Boundary and initial conditions.

\begin{tabular}{ll} 
Boundary & Condition \\
\hline Initial concentration & $a=1$ \\
Bulk solution concentration & $a=1$ \\
Concentration at generator electrode & $a=0$ \\
Concentration at collector electrode & $b=0$ \\
Boundary condition at generator and collector electrodes & $\frac{\partial a}{\partial Z}=-D_{\mathrm{r}} \frac{\partial b}{\partial Z}$ \\
Axis of symmetry and insulator surface & $\frac{\partial a}{\partial R}=0$ \\
Diffusion domain border & $\frac{\partial a}{\partial R}=0$ \\
Insulator surface & $\frac{\partial a}{\partial Z}=0$
\end{tabular}


Table 3: Steady state current and time of diffusional independence on disks electrodes and generator-collector electrodes* within the array.

\begin{tabular}{rrrr|rrr}
\hline \multicolumn{4}{c|}{ microdisk } & \multicolumn{3}{c}{ generator-collector } \\
\hline$\frac{d}{r_{0}}$ & $r_{0}=100 \mathrm{~nm}$ & $r_{0}=1 \mu \mathrm{m}$ & $r_{0}=10 \mu \mathrm{m}$ & $r_{0}=100 \mathrm{~nm}$ & $r_{0}=1 \mu \mathrm{m}$ & $r_{0}=10 \mu \mathrm{m}$ \\
5 & $6 \mathrm{E}-5 ; 1.5 \mathrm{E}-7 * *$ & $6 \mathrm{E}-3 ; 1.5 \mathrm{E}-8$ & $0.6 ; 1.5 \mathrm{E}-9$ & $\infty ; 3 \mathrm{E}-7$ & $\infty ; 3 \mathrm{E}-8$ & $\infty ; 3 \mathrm{E}-9$ \\
10 & $6 \mathrm{E}-4 ; 3.9 \mathrm{E}-8$ & $0.04 ; 3.9 \mathrm{E}-9$ & $4 ; 3.9 \mathrm{E}-10$ & $\infty ; 7.4 \mathrm{E}-8$ & $\infty ; 7.4 \mathrm{E}-9$ & $\infty ; 7.4 \mathrm{E}-10$ \\
50 & $0.055 ; 1.5 \mathrm{E}-9$ & $5.5 ; 1.5 \mathrm{E}-10$ & $550 ; 1.5 \mathrm{E}-11$ & $\infty ; 3 \mathrm{E}-9$ & $\infty ; 3 \mathrm{E}-10$ & $\infty ; 3 \mathrm{E}-11$ \\
\hline
\end{tabular}

Table 4: * Geometry of the generator collector electrode is definite by the next parameters $L_{\mathrm{cyl}}=0.1, R_{1}=R_{\max }$, area of the electrode array is $1 \mathrm{~mm}^{2}$. ** The first number is time, $\mathrm{s}$ of diffusional independence of electrodes within the array, the second number is the values of current, A from the microelectrode array. 


\section{Figures}

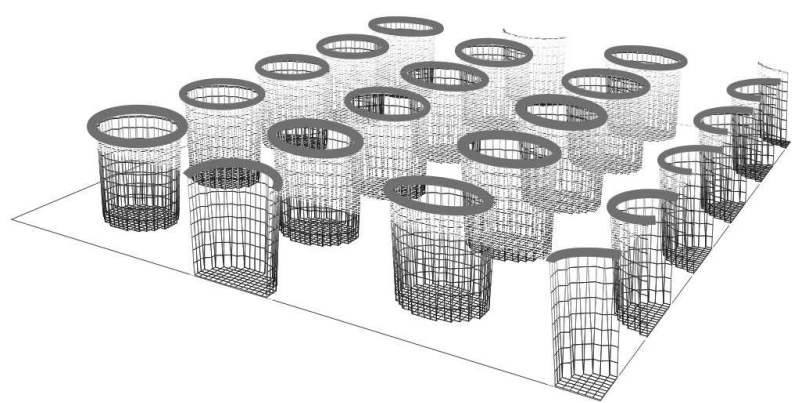

Figure 1: Schematic diagram of the electrode surface 
(a)

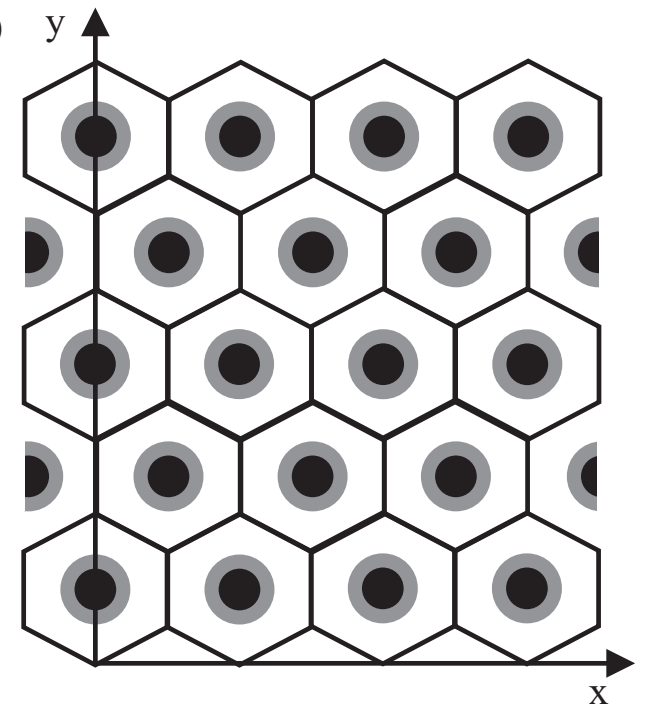

(b)

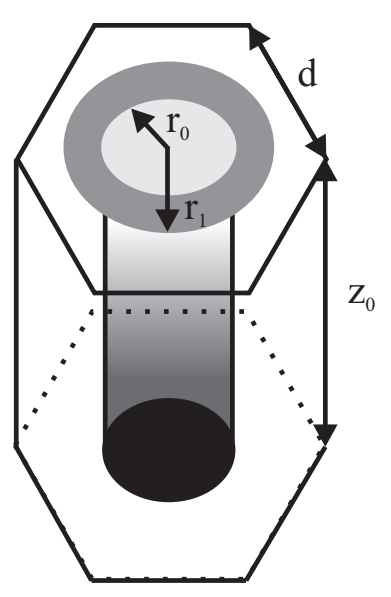

(c)

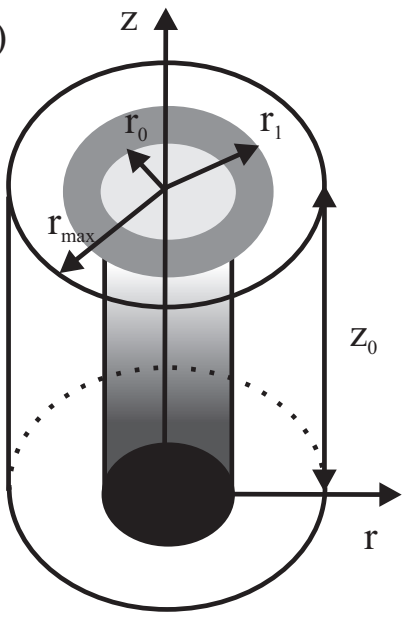

Figure 2: (a)Schematic diagram of the unit cell for an array of ring-recessed electrodes; (b) single unit cell in Cartesian coordinates; (c) equivalent diffusion domain in cylindrical coordinates 


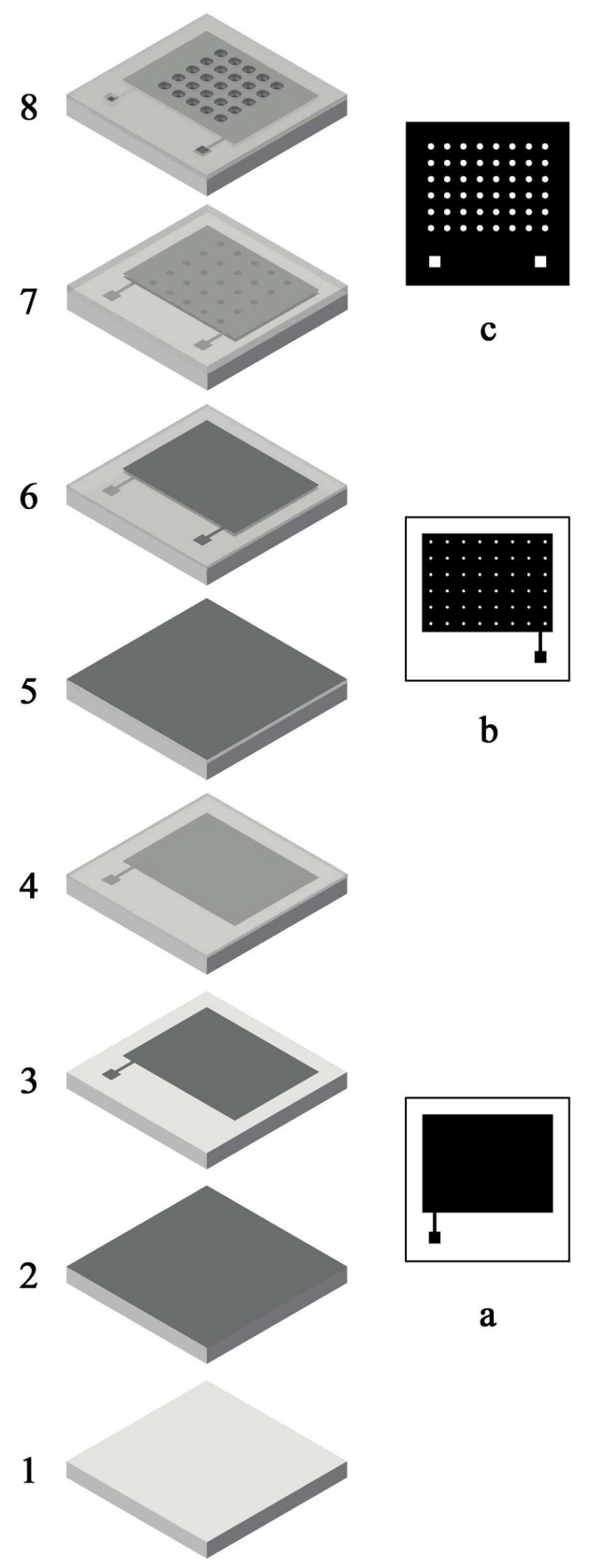

Figure 3: Diagrammatic representation of main steps in the fabrication process (1-8) and mask types used (a-c). 


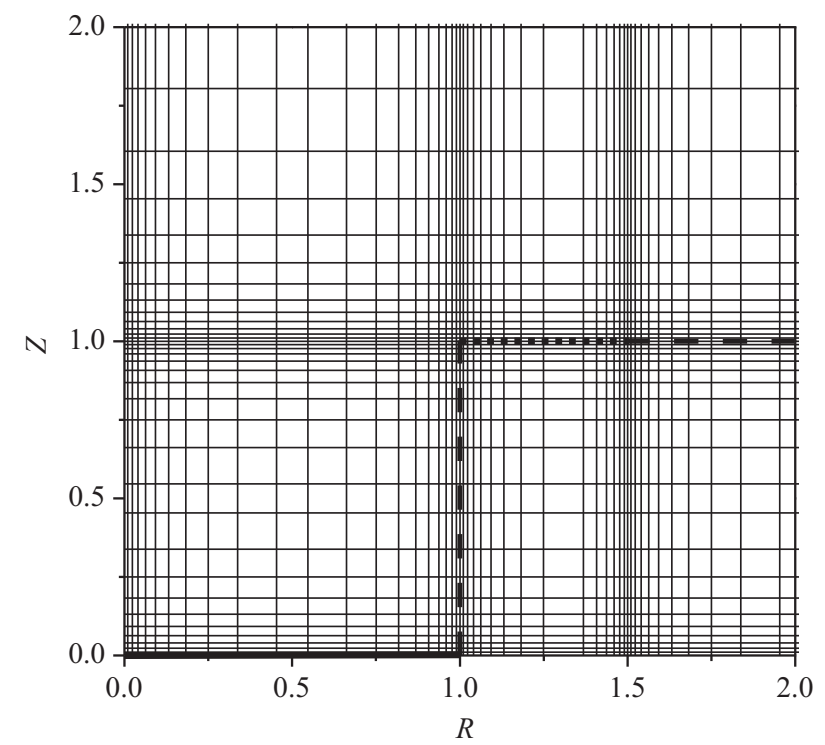

Figure 4: Expanding grid used in this work (some lines excluded for clarity). The generator electrode is shown in solid black, the collector electrode is in black squares and the insulator is in black dashes 


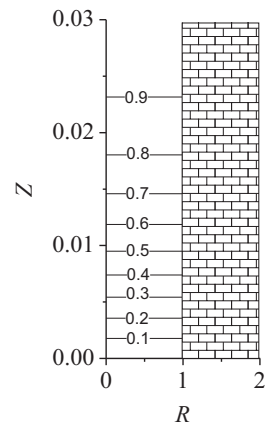

b)

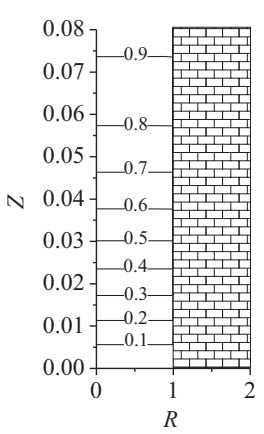

c)

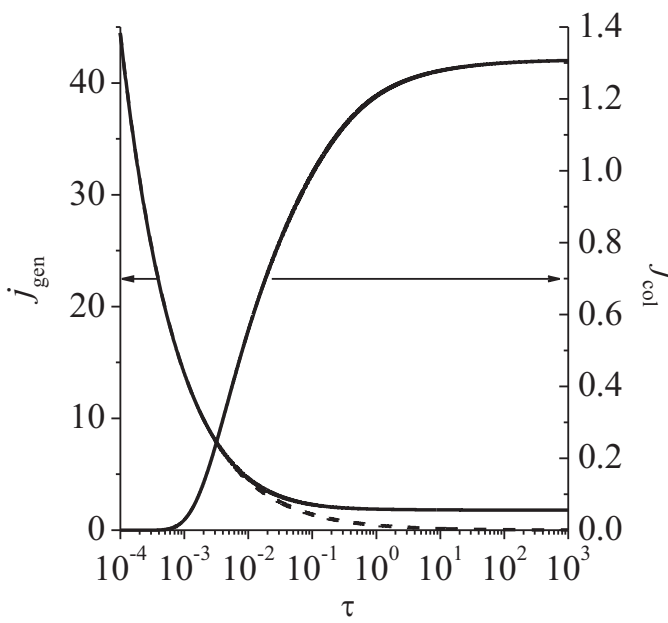

a)

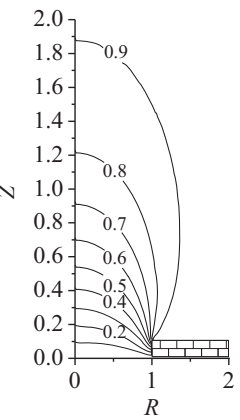

e)

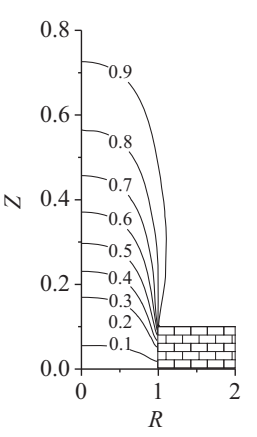

d)

Figure 5: a) Calculated dimensionless current at generator and collector electrodes (solid lines) versus dimensionless time in the potential step experiment, dashed line represents current transient given by the Cottrell equation, $L_{\mathrm{cyl}}=0.1$ b), c), d), e) Concentration profile at $\tau=10^{-4}, 10^{-3}, 0.1,100$ correspondingly 


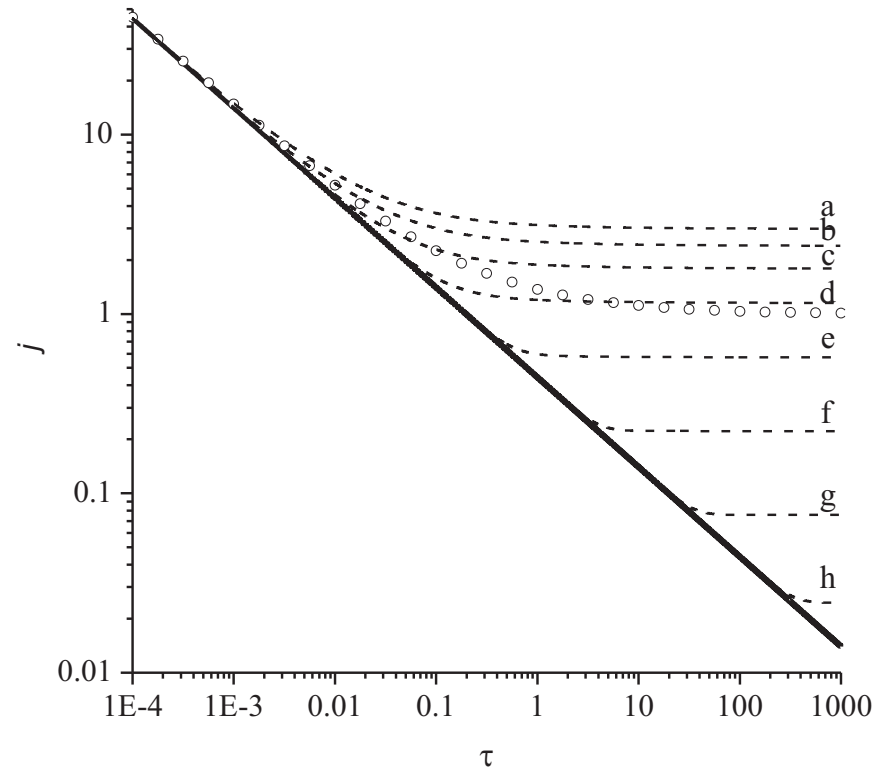

a)

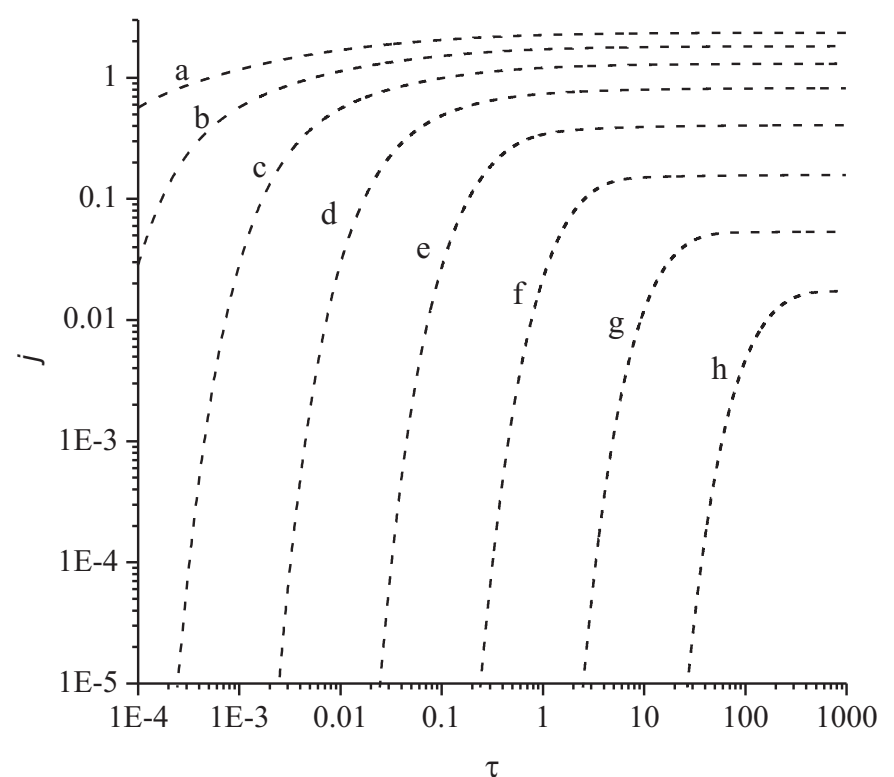

b)

Figure 6: Absolute value of calculated dimensionless current at generator a) and collector b) versus dimensionless time in the potential step experiment, single electrode, $R_{1}=1.1$. Solid and dotes lines corresponds to current at macro- and microelectrodes. Dashed lines represents current at recessed generator-collector electrodes with $L_{\text {cyl }}=0.01 \mathrm{a}, 0.032 \mathrm{~b}, 0.1 \mathrm{c}, 0.32 \mathrm{~d}, 1 \mathrm{e}$, $3.2 \mathrm{f}, 10 \mathrm{~g}, 32 \mathrm{~h}$. 


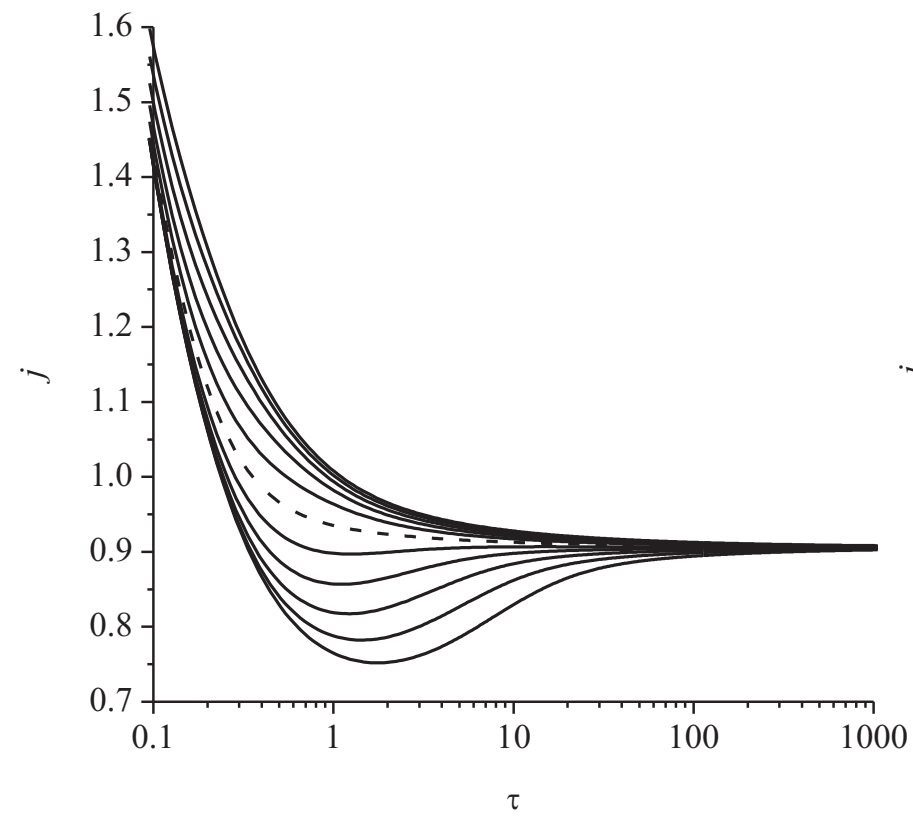

a)

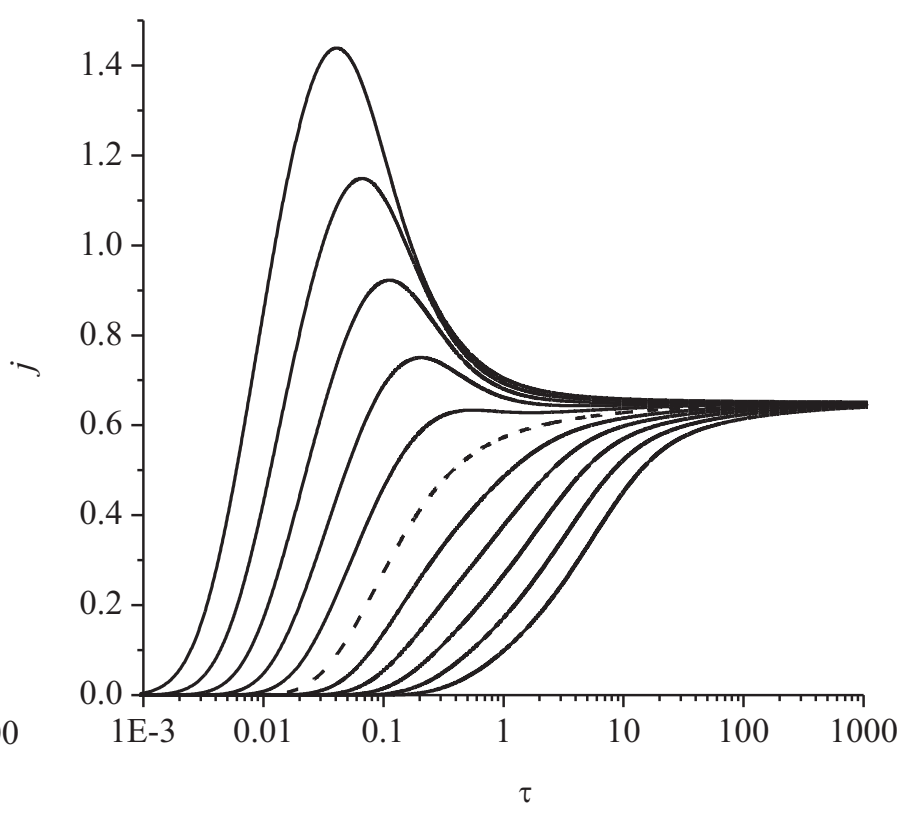

b)

Figure 7: Absolute value of calculated dimensionless current at generator a) and collector b) electrodes versus dimensionless time in the potential step experiment, single electrode, $R_{1}=1.1$, $L_{\text {cyl }}=0.5$. Dashed line represents case of equal diffusion coefficients, solid lines represents results of modeling with unequal diffusion coefficients. $D_{\mathrm{r}}$ have values of $0.1,0.16,0.25,0.40$, $0.63,1.6,2.5,4.0,6.3,10$ from the lowest to the highest line. 


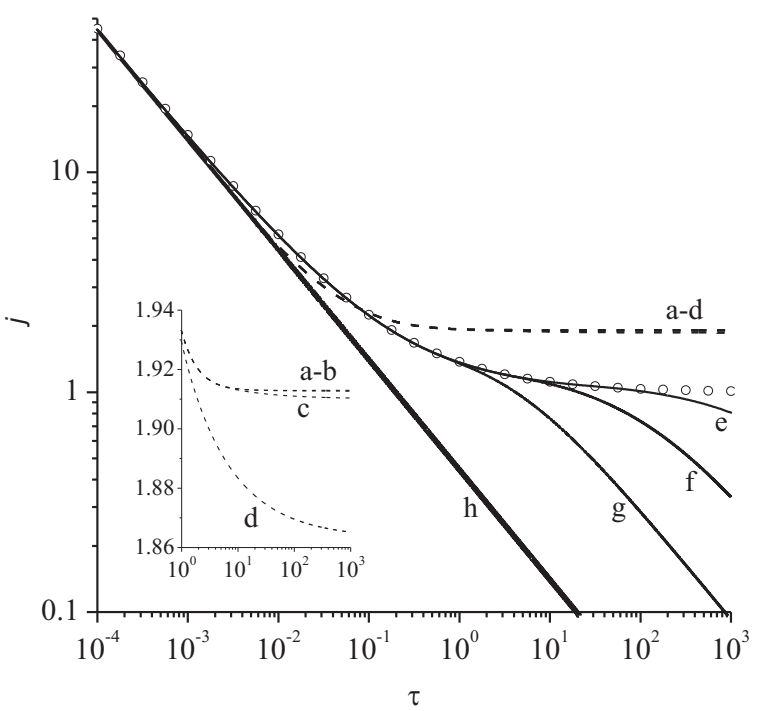

a)

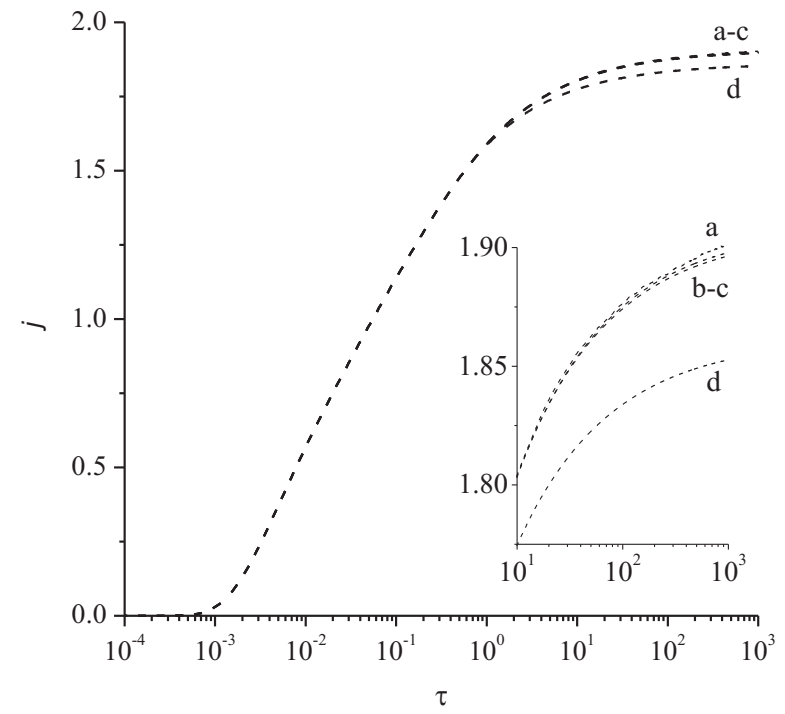

b)

Figure 8: Influence of diffusional domain radius on current transients. $R_{1}=1.1$ and $L_{\text {cyl }}=0.1$.

a) circles corresponds to single microelectrode, solid line h corresponds to macroelectrode, solid lines g, f and e corresponds to microelectrodes in the array with $R_{\max }=5,10$ and 13 correspondingly, dashed lines a and b, c, d represents current on generator at single recessed generator-collector device and the array of generator-collector device with $R_{\max }=10,5$ and 2 correspondingly. b) dashed lines a and b, c, d represents current on collector at single recessed generator-collector device and the array of generator-collector device with $R_{\max }=10,5$ and 2 correspondingly. 

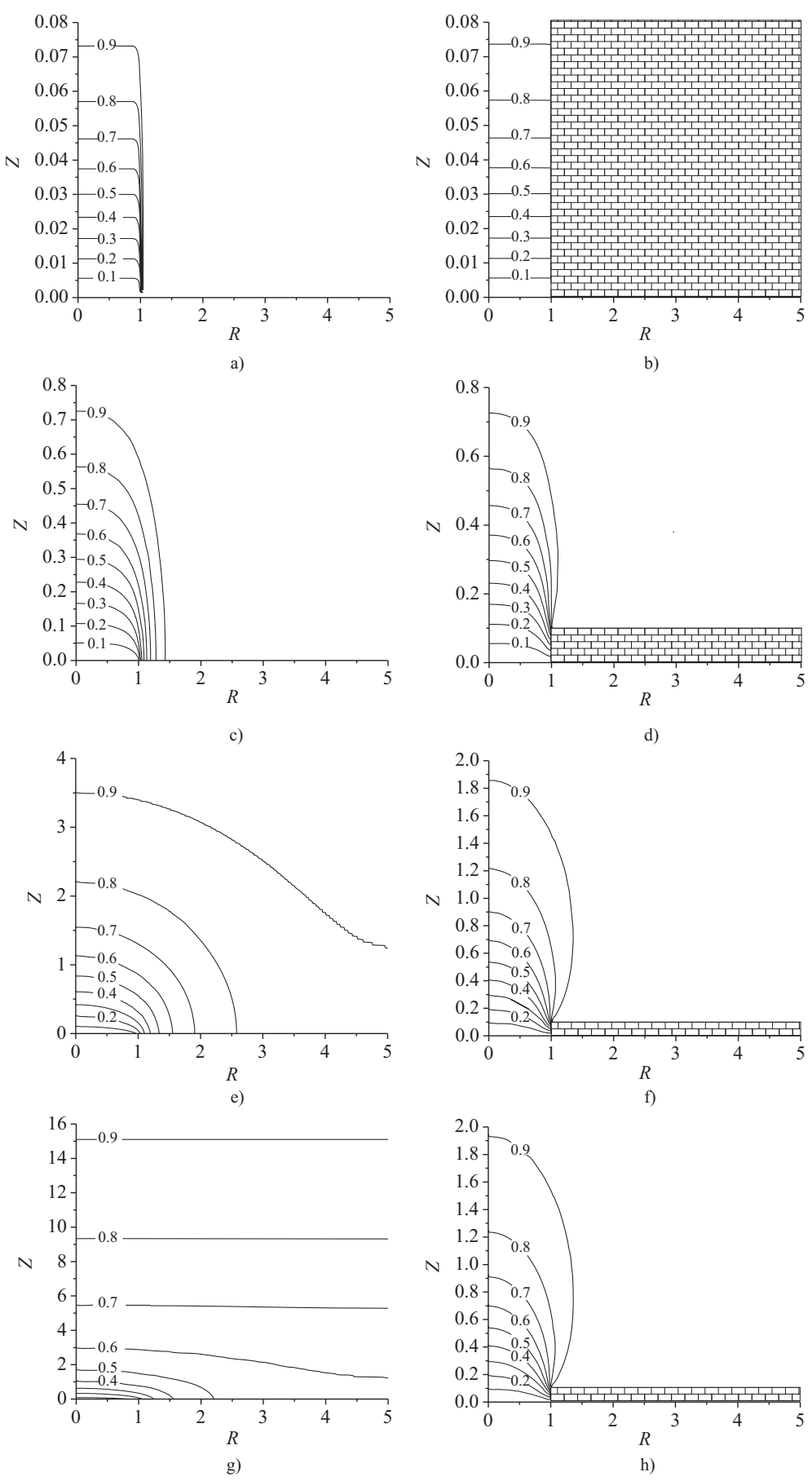

Figure 9: Simulated concentrations profiles, at array of microelectrodes (a), c), e), g)) and recessed generator-collector (b), d), f), h)): $\tau=0.001$ a) and b), $\tau=0.1$ c) and d), $\tau=10$ e) and $\mathrm{f}), \tau=100 \mathrm{~g}$ ) and $\mathrm{h}$ ) 


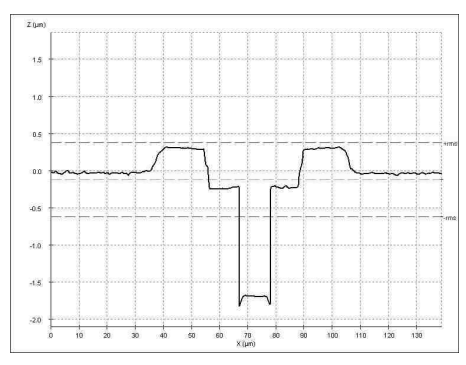

a)

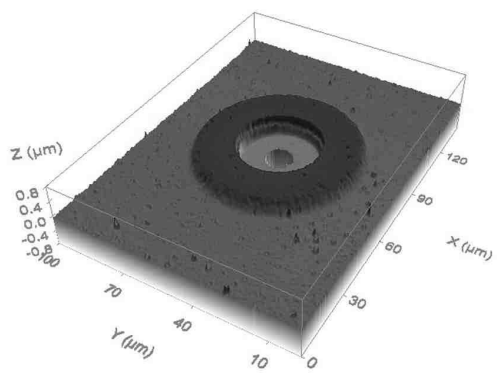

b)

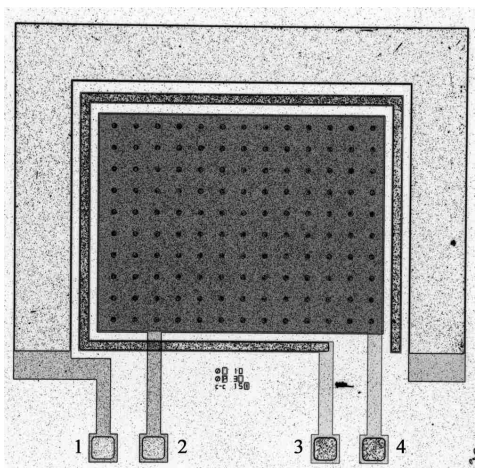

c)

Figure 10: a) Perfolimetry profile. b) Confocal microscopy image showing, in detail, a typical ring-recessed disc electrode in an array. The disc diameter, which is also the ring inner diameter, is 10 microns. The ring outer diameter is 30 microns. The disc recess height is approximately 1.6 microns c) Optical microscope image of the chip type used in this work. The chip features 4 electrodes. From left to right, 1 is a large electrode that can be used as auxiliary electrode. 2 corresponds to the recessed microdiscs, 3 is a thin microband intended for use as quasi-reference electrode and 4 connects to the top microring electrodes. 


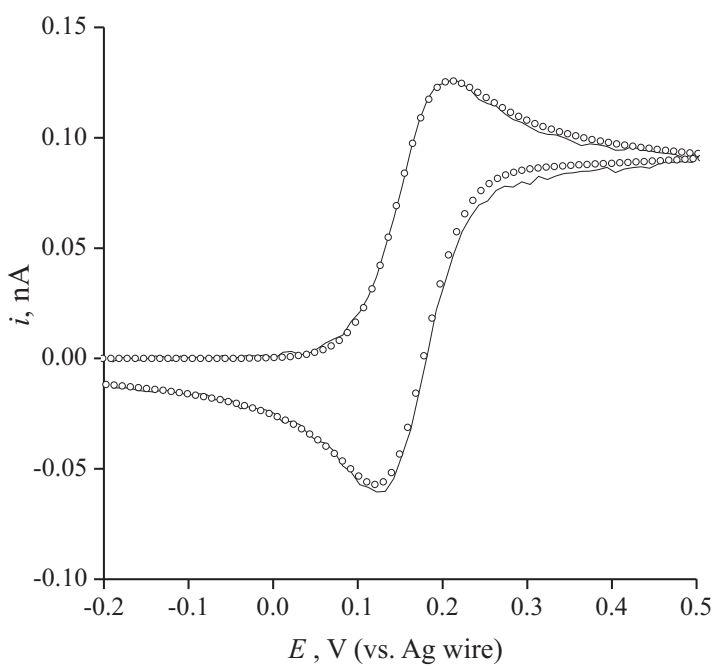

a)

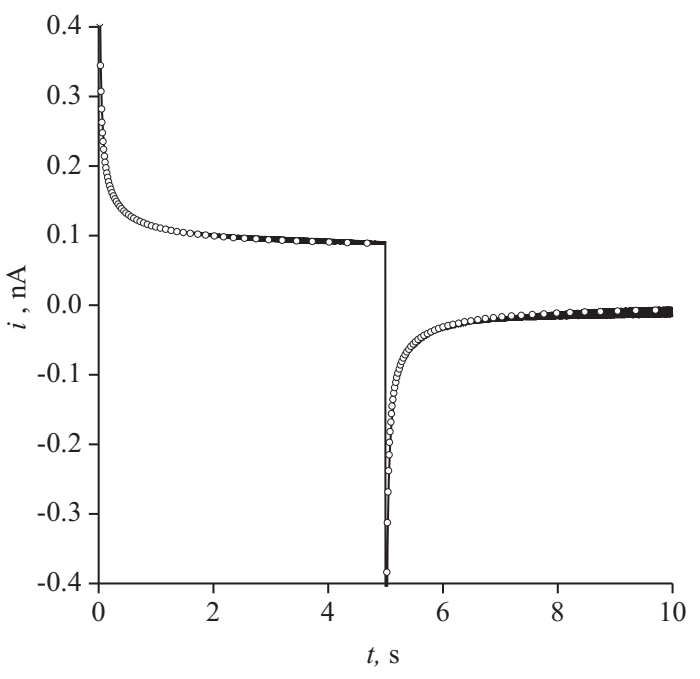

b)

Figure 11: a) Cyclic voltammogram of ferrocene in $\left[\mathrm{N}_{6,2,2,2}\right]\left[\mathrm{NTf}_{2}\right]$ at $10 \mu \mathrm{m}$ Pt electrode vs Ag wire at $100 \mathrm{mV} \mathrm{s}^{-} 1$. b)Double-step chronoamperometry of $\mathrm{Fc}$ in $\left[\mathrm{N}_{6,2,2,2}\right]\left[\mathrm{NTf}_{2}\right]$ at microdisk platinum electrode $(10 \mu \mathrm{m}$ diameter $)$ vs silver wire for duration $5 \mathrm{~s}$ at sample time $0.01 \mathrm{~s}$. Experimental data is shown as a line and theoretical fit is plotted as cycles. 


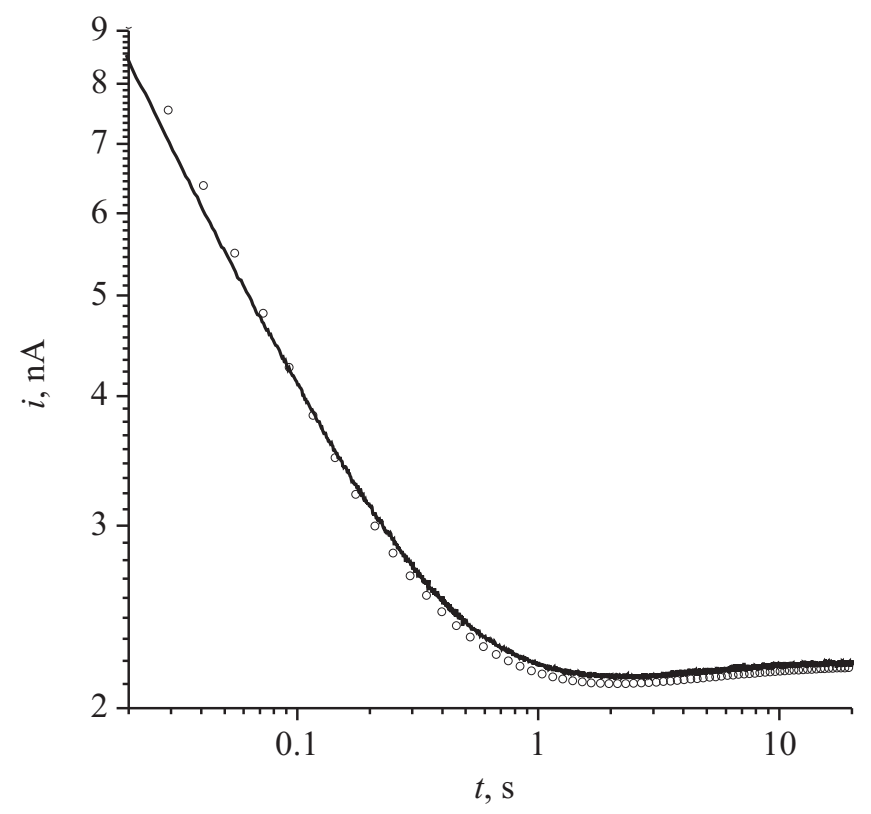

a)

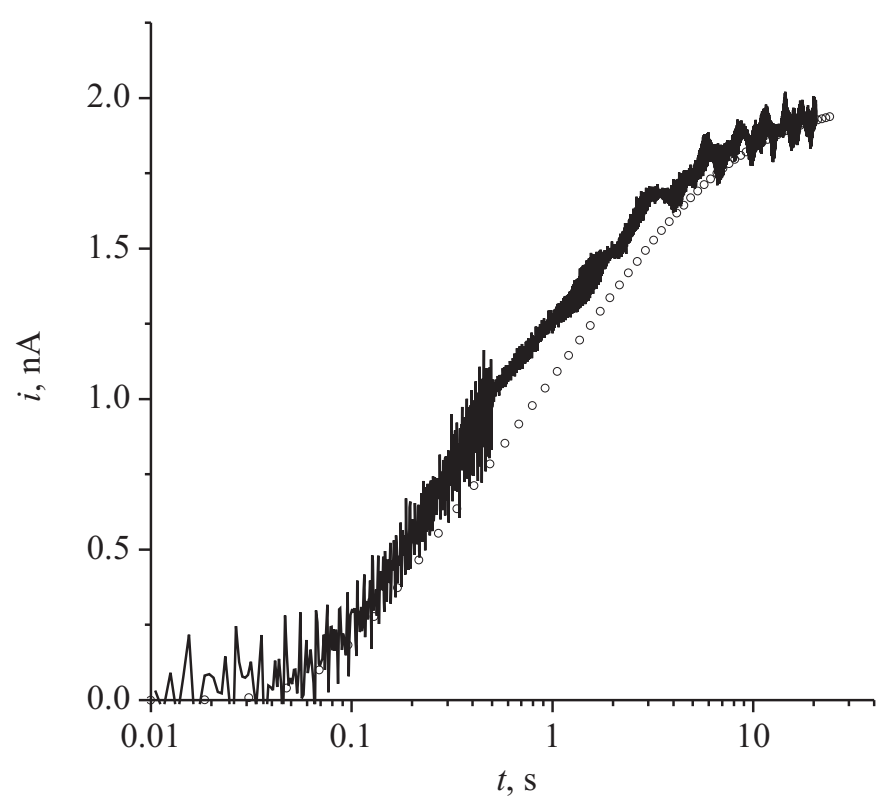

b)

Figure 12: Bipotentiostat chronoamperometry of $\mathrm{Fc}_{\mathrm{c}}$ in $\left[\mathrm{N}_{6,2,2,2}\right]\left[\mathrm{NTf}_{2}\right]$ at ring-recessed disk electrode array for a) generator electrode and b) collector electrode, experimental data is shown as a line and theoretical fit is plotted as cycles. $\mathrm{D}_{\mathrm{Fc}}=10.0 \times 10^{-12}, \mathrm{D}_{\mathrm{Fc}^{+}}=5.50 \times 10^{-12}$ 